\title{
Optimization of Machining Parameters of SiC Single Crystal Cut by Diamond Wire Saw
}

\author{
Lun $\mathrm{Li}^{1,2, a}$, Shaodong Yang ${ }^{1,2}$ and Jishun $\mathrm{Li}^{1,2}$ \\ ${ }^{1}$ School of Mechatronics Engineering, Henan University of Science and Technology , 471003 Luoyang, China \\ ${ }^{2}$ Henan Key Laboratory for Machinery Design and Transmission System, Henan University of Science and Technology, 471003 \\ Luoyang, China
}

\begin{abstract}
For the process of optimizing the process parameters in the process of cutting the hard and brittle materials such as single-crystal $\mathrm{SiC}$ by diamond wire saw, the process parameters such as wire saw speed, workpiece feed speed and workpiece rotation speed are the design variables, and the sawing force and surface roughness are the processing targets by orthogonal experimental design. The grey system theory is introduced to optimize the multi-objective cutting process. According to the experimental data, the grey correlation resolution coefficient is determined. The significant relationship between the processing parameters and the target characteristics is analyzed. The optimal combination of process parameters under multi-target conditions is obtained, namely the workpiece feed rate is $0.025 \mathrm{~mm} / \mathrm{min}$, the wire saw speed is $1.6 \mathrm{~m} / \mathrm{s}$, and the workpiece rotating speed is $16 \mathrm{r} / \mathrm{min}$.
\end{abstract}

\section{Introduction}

$\mathrm{SiC}$, as the third generation of the main semiconductor material, has high Young's modulus, high hardness, high radiation resistance at high temperatures, good corrosion resistance, and a unique wide bandgap, high critical breakdown field strength and high thermal conductivity that make it widely used in aeronautics and astronautics, optoelectronics integration and microelectronics [1,2].

The fixed abrasive diamond wire saw is widely used in the cutting and processing of hard and brittle materials because of its characteristics such as narrow slit, low material loss, low pollution, high cutting efficiency and suitable for processing large-diameter materials. The cutting process is the first process in the single wafer production process, and the production cost accounts for more than $50 \%$ of the total processing cost. The processing quality and processing efficiency directly affect the efficiency and cost of subsequent processing. Therefore, it is very important to analyze the influence of process parameters on the sawing force and surface roughness in the cutting process, and to find the optimal process parameters that meet the processing requirements and improve the processing efficiency $[3,4]$.

In recent years, many domestic and foreign scholars have conducted a lot of research work on the optimization of the process parameters of diamond wire saws for cutting hard and brittle materials. Clark [3] used diamond wire saws to perform cutting experiment on ceramics, analyzed the effects of processing parameters on sawing force, force ratio, and surface roughness. Craig [4] performed single-crystal $\mathrm{SiC}$ cutting tests on a sway saw, and studied the effects of workpiece feed speed and sawing wire sway frequency on wafer surface quality. Gao [5] used self-made electroplated diamond wire saws to study the influence of process parameters and the cutting fluid on the surface and subsurface of singlecrystal Si cutting. During the cutting process of SiC wafers using electroplated diamond wire saws, the cutting time is relatively long and the number of experiments is limited. In this paper, the grey system theory is used to analyze the influence of the processing parameters on the sawing force and the surface quality, as well as to obtain the optimal parameter combination of improving the surface quality and improving the life of the wire saw.

\section{Experimental scheme analysis and design}

The experiment uses a modified WXD170 type reciprocating diamond wire saw rotary cutting machine. The $\mathrm{X}, \mathrm{Y}$ axis moving platform stroke is $120 \mathrm{~mm}$, the feed speed can be steplessly speed regulation between $0.005 \sim 18 \mathrm{~mm} / \mathrm{min}$, the table rotation speed between $0 \sim 36 \mathrm{r} / \mathrm{min}$. The point-contact rotary cutting between the wire saw and the workpiece can be achieved, and tap water is used as a cooling liquid for the cutting process. The sawing force measuring device adopts Gama SI-322.5 multi-axis force/torque sensor of American ATI company and 9105-MIUSB 16-channel data acquisition system of American NI company to collect sawing force in real time. The surface roughness was measured with a Leica DCM 3D confocal microscope and measured from the center of the $\mathrm{SiC}$ section in six different directions.

In the process of cutting $\mathrm{SiC}$ single crystal by diamond wire saw, the precision of the machine tool, the contact

* Corresponding author: ${ }^{\text {a lilunxn@ } @ 163 . c o m}$ 
angle of the wire saw, the tension of the wire saw, the wear and the grain size of the diamond wire saw and the processing parameters have a complex effect on the sawing force and the surface roughness. During the reciprocating cutting process of the wire saw, the high speed of the wire saw causes the poor vibration stability of the machine tool, which is not conducive to the change of the wire saw when the wire saw is too low, so the range of the value is $1.3-1.9 \mathrm{~m} / \mathrm{s}$. When the feed speed is more than $0.08 \mathrm{~mm} / \mathrm{min}$, the wire saw broken wire is frequent and $\mathrm{SiC}$ slide appears, which affects the surface quality and processing efficiency of the workpiece, so the range of the feed speed is $0.025-0.08 \mathrm{~mm} / \mathrm{min}$. The rotation of the workpiece cannot only keep the contact length between the workpiece and the wire saw in the cutting process, but also improve the machining efficiency and improve the machining accuracy. When the speed is too large, the inertia impact of the online saw is more obvious, so the range of its value is $8-16 \mathrm{r} / \mathrm{min}$.

In this paper, wire saw sawing speed(A), workpiece feed speed(B) and workpiece rotation speed(C) are taken as experimental parameters (as shown in Table 1). The experimental design of the $\mathrm{L}_{9}\left(3^{4}\right)$ orthogonal table was used to measure the sawing force $(F)$ and wafer surface roughness $(\mathrm{Ra})$ under different processing parameter combinations. The experimental scheme and experimental results are shown in Table 2.

Table 1. Parameters and levels of orthogonal experimental design.

\begin{tabular}{|c|c|c|c|}
\hline Level & $\mathbf{A} /\left(\mathbf{m} \cdot \mathbf{s}^{\mathbf{- 1}}\right)$ & $\mathbf{B} /\left(\mathbf{m m} \cdot \mathbf{m i n}^{\mathbf{- 1}}\right)$ & $\mathbf{C} /\left(\mathbf{r} \cdot \mathbf{m i n}^{\mathbf{- 1}}\right)$ \\
\hline 1 & 1.3 & 0.025 & 8 \\
\hline 2 & 1.6 & 0.05 & 12 \\
\hline 3 & 1.9 & 0.08 & 16 \\
\hline
\end{tabular}

Table 2. Experimental results of $\mathrm{SiC}$ single crystal cutting.

\begin{tabular}{|c|c|c|c|c|c|}
\hline \multirow{2}{*}{ No. } & \multicolumn{3}{|c|}{ Parameters } & \multicolumn{2}{c|}{ Experimental Result } \\
\cline { 2 - 6 } & $\mathrm{A}$ & $\mathrm{B}$ & $\mathrm{C}$ & $\mathrm{F}$ & $\mathrm{Ra}$ \\
\hline 1 & 1 & 1 & 1 & 5.34 & 0.842 \\
\hline 2 & 1 & 2 & 2 & 6.91 & 1.103 \\
\hline 3 & 1 & 3 & 3 & 8.16 & 1.152 \\
\hline 4 & 2 & 1 & 2 & 4.58 & 0.684 \\
\hline 5 & 2 & 2 & 3 & 6.28 & 0.943 \\
\hline 6 & 2 & 3 & 1 & 7.47 & 0.980 \\
\hline 7 & 3 & 1 & 3 & 5.14 & 0.815 \\
\hline 8 & 3 & 2 & 1 & 6.74 & 0.992 \\
\hline 9 & 3 & 3 & 2 & 7.68 & 1.016 \\
\hline
\end{tabular}

\section{Grey relational analysis theory and calculation}

Grey relational analysis is a very important part of the systematic analysis in the grey system theory. The basic idea is to express the approximate relation between sequences according to the similarity degree of the geometric shape of different sequence curves in the sequence, that is, the approximate relation between different sequences is expressed by the method of linear difference. It can compensate for the lack of information in the analysis of variance, regression analysis [6-7]. In the case of no large sample or regular sample, the grey correlation degree of the wire saw cutting speed, the workpiece feed speed and the rotation speed to F, Ra ,can be given in the process of cutting the wire saw.

In the system, due to the differences in the units, meanings of each sequence, each sequence must be properly processed before data analysis, and it can be converted into dimensionless data of the same order of magnitude through the action of operators. A set of data under each process target in the test results in Table 2 is the original data sequence, that is, the original data sequence of $\mathrm{x}_{\mathrm{i}}(\mathrm{k})$ representing the $\mathrm{F}$ and $\mathrm{Ra}$.

First, an interval valued operator equation (1) is used to normalize the original data series.

$$
\mathrm{y}_{\mathrm{i}}(\mathrm{k})=\frac{\mathrm{x}_{\mathrm{i}}(\mathrm{k})-\min \mathrm{x}_{\mathrm{i}}(\mathrm{k})}{\max \mathrm{x}_{\mathrm{i}}(\mathrm{k})-\min \mathrm{x}_{\mathrm{i}}(\mathrm{k})}
$$

In the equation $(1): \mathrm{i}=1,2, \ldots, \mathrm{n} ; \mathrm{k}=1,2, \ldots, \mathrm{m}$; Where $\mathrm{n}$ is the number of data sequence parameters; $m$ is the number of experiments; $\mathrm{x}_{\mathrm{i}}(\mathrm{k})$ is the $\mathrm{kth}$ experiment under the $\mathrm{i}$-th index, and $\mathrm{y}_{\mathrm{i}}(\mathrm{k})$ is the data sequence obtained after data preprocessing.

Calculating the grey correlation coefficient $\xi_{\mathrm{i}}(\mathrm{k})$ of the comparison sequence $\mathrm{y}_{\mathrm{i}}(\mathrm{k})$ relative to the reference sequence $\mathrm{y}_{0}(\mathrm{k})$ using equation (2) (as shown in Table 4), the sawing force and the surface roughness are taken as the minimum : $\mathrm{y}_{0}(\mathrm{k})=0$.

$$
\xi_{i}(k)=\frac{\min _{i} \min _{k}\left|\mathrm{y}_{i}(k)-\mathrm{y}_{0}(k)\right|+\mu \max _{i} \max _{k}\left|\mathrm{y}_{i}(k)-\mathrm{y}_{0}(k)\right|}{\left|\mathrm{y}_{i}(k)-\mathrm{y}_{0}(k)\right|+\mu \max _{i} \max _{k}\left|\mathrm{y}_{i}(k)-\mathrm{y}_{0}(k)\right|}
$$

In the equation: $\mu$ is grey correlation resolution coefficient. Its value can be obtained according to the following equation. Setting $\Delta_{\mathrm{v}}$ that is the mean of all absolute differences and

$$
\begin{gathered}
\varepsilon=\Delta_{\mathrm{v}} / \Delta_{\max } \quad\left(\Delta_{\max }=1\right) \\
\Delta_{\mathrm{v}}=1 /(m n) \sum_{i=1}^{m} \sum_{k=1}^{n}\left|\mathrm{y}_{i}(k)-\mathrm{y}_{0}(k)\right|
\end{gathered}
$$

When $\Delta_{\max } \leq 3 \Delta_{\mathrm{v}}, 1.5 \varepsilon<\mu \leq 2 \varepsilon$;

When $\Delta_{\max } \geq 3 \Delta_{\mathrm{v}}, \varepsilon<\mu \leq 1.5 \varepsilon$;

Table 3. Absolute difference of each sequence.

\begin{tabular}{|c|c|c|}
\hline \multirow{2}{*}{ No. } & \multicolumn{2}{|c|}{ Absolute Difference } \\
\cline { 2 - 3 } & $\mathrm{F}$ & $\mathrm{Ra}$ \\
\hline 1 & 0.2123 & 0.3376 \\
\hline 2 & 0.6508 & 0.8953 \\
\hline 3 & 1.0000 & 1.0000 \\
\hline 4 & 0 & 0 \\
\hline 5 & 0.4749 & 0.5534 \\
\hline 6 & 0.8073 & 0.6325 \\
\hline 7 & 0.1564 & 0.2799 \\
\hline 8 & 0.6034 & 0.6581 \\
\hline 9 & 0.8659 & 0.7094 \\
\hline
\end{tabular}


The absolute difference of the comparison sequence of each target with respect to the reference sequence( as shown in Table 3 ) is brought into the equation (4):

$\Delta_{\mathrm{v}}=0.5465, \mu=0.85$.

The values in Table 4 are substituted into Equation (7) to calculate the grey correlation coefficient, and the grey correlation degree $\gamma(\mathrm{k})$ is calculated (as shown in Table 4).

$$
\gamma(k)=\frac{1}{2} \sum_{i=1}^{\mathrm{n}} \sum_{k=1}^{\mathrm{m}} \xi_{i}(k)
$$

Table 4. Grey correlation coefficient and grey correlation degree of each sequence.

\begin{tabular}{|c|c|c|c|}
\hline \multirow{2}{*}{ No. } & \multicolumn{2}{|c|}{$\begin{array}{c}\text { Grey Correlation } \\
\text { Coefficient }\end{array}$} & \multirow{2}{*}{$\begin{array}{c}\text { Grey Correlation } \\
\text { Degree }\end{array}$} \\
\cline { 2 - 3 } & $\mathrm{F}$ & $\mathrm{Ra}$ & \\
\hline 1 & 0.8002 & 0.7157 & 0.7579 \\
\hline 2 & 0.5664 & 0.4870 & 0.5267 \\
\hline 3 & 0.4595 & 0.4595 & 0.4595 \\
\hline 4 & 1.0000 & 1.0000 & 1.0000 \\
\hline 5 & 0.6416 & 0.6057 & 0.6236 \\
\hline 6 & 0.5129 & 0.5734 & 0.5431 \\
\hline 7 & 0.8446 & 0.7523 & 0.7984 \\
\hline 8 & 0.5849 & 0.5636 & 0.5742 \\
\hline 9 & 0.4954 & 0.5451 & 0.5202 \\
\hline
\end{tabular}

\section{Grey relational analysis parameter optimization}

\subsection{Single-objective grey relational analysis and parameter optimization}

According to the concept of grey correlation degree, the process parameters with the maximum difference in the mean difference of correlation coefficient have the greatest influence on the target. The maximum association coefficient of the different parameters of each level makes up the optimal combination of the target.

Table 5. The average grey correlation coefficient for different levels of various parameters of $\mathrm{F}$.

\begin{tabular}{|c|c|c|c|c|}
\hline \multirow{2}{*}{ Parameters } & \multicolumn{3}{|c|}{$\begin{array}{c}\text { Average Grey Correlation } \\
\text { Coefficient }\end{array}$} & \multirow{2}{*}{ Range } \\
\cline { 2 - 4 } & Level 1 & Level 2 & Level 3 & \\
\hline A & 0.6087 & 0.7182 & 0.6416 & 0.1095 \\
\hline B & 0.8816 & 0.5976 & 0.4892 & 0.3924 \\
\hline C & 0.6326 & 0.6872 & 0.6485 & 0.0546 \\
\hline
\end{tabular}

Table 6. The average grey correlation coefficient for different levels of various parameters of Ra.

\begin{tabular}{|c|c|c|c|c|}
\hline \multirow{2}{*}{ Parameters } & \multicolumn{3}{|c|}{$\begin{array}{c}\text { Average Grey Correlation } \\
\text { Coefficient }\end{array}$} & \multirow{2}{*}{ Range } \\
\cline { 2 - 4 } & Level 1 & Level 2 & Level 3 & \\
\hline A & 0.5541 & 0.7263 & 0.620 & 0.1722 \\
\hline B & 0.8227 & 0.5521 & 0.5260 & 0.2967 \\
\hline
\end{tabular}

\begin{tabular}{|l|l|l|l|l|}
\hline $\mathrm{C}$ & 0.6176 & 0.6774 & 0.6058 & 0.0716 \\
\hline
\end{tabular}

Based on Table 4, the average grey correlation coefficient of sawing force and surface roughness is calculated, and the single objective optimization of $\mathrm{F}$ and $\mathrm{Ra}$ is carried out (as shown in Table 5 and Table 6). It can be seen from Table 5 that the order of influence on sawing force from big to small is: workpiece feed speed, wire saw speed, workpiece rotation speed; the best combination is $\mathrm{A} 3 \mathrm{~B} 1 \mathrm{C} 2$. It can be seen from Table 6 that the order of the influence of surface roughness from big to small is: workpiece feed speed,wire saw speed, workpiece rotation speed; the best combination is A2B1C2.

\subsection{Multi-objective grey relational analysis and parameter optimization}

In the orthogonal test design, each process parameter does not affect each other, there is a grey correlation coefficient difference between different levels of the process parameters, which reflects the impact of different levels of each process parameter on the comprehensive multiobjective. The grey correlation degree between the sawing force and the surface roughness is calculated according to the grey correlation coefficient of different levels of each parameter in Table 4. It can be seen from Table 7 that the degree of influence on the cutting quality and effect is in the following order: workpiece feed speed, wire saw speed, workpiece rotation speed.

Table 7. The average grey correlation of each parameter in different level.

\begin{tabular}{|c|c|c|c|c|}
\hline \multirow{2}{*}{ Parameters } & \multicolumn{3}{|c|}{ Average Grey Correlation } & \multirow{2}{*}{ Degree } \\
\cline { 2 - 4 } & Level 1 & Level 2 & Level 3 & \\
\hline A & 0.5814 & 0.7222 & 0.6310 & 0.1408 \\
\hline B & 0.8521 & 0.5748 & 0.5076 & 0.3484 \\
\hline C & 0.6251 & 0.6823 & 0.6272 & 0.0572 \\
\hline
\end{tabular}

The maximum value of the average grey correlation of the workpiece feed rate is on the level 1, and the value on the level 3 is the smallest. This shows that as the workpiece feed speed increases, the contact arc length between the workpiece and the wire saw increases, and the wire saw bends seriously, increasing the cutting depth of the wire saw and increasing the sawing force of the wire saw. Because the cutting fluid cannot enter the cutting area in the continuous cutting process, the chips and detached abrasive grains cannot be eliminated in time. In a narrow cutting area, cracks are easily generated and expanded, and the workpiece processing quality is drastically reduced. The tangential friction force of the wire saw increases, the normal force increases, and the abrasive particles fall off quickly, and the wire breakage is prone to occur, and the life of the wire saw is reduced.

The average value of the grey correlation between the wire saw speed and the workpiece rotation speed is at the level 2, which means that reducing or increasing the wire saw speed and the workpiece rotation speed can increase 
the sawing force and the surface roughness reduces the life of the wire saw. Combining the combination of the maximum values of the correlation degrees in the various process parameters in Table 7 to form the optimal combination of multiple process objectives, namely, the speed of the sawing machine is $1.6 \mathrm{~m} / \mathrm{s}$, the workpiece feed rate is $0.025 \mathrm{~mm} / \mathrm{min}$, and the workpiece rotation speed is $12 \mathrm{r} / \mathrm{min}$. In the process of the combination, the contact arc between the wire saw and the workpiece can be kept in a reasonable range, reducing the normal force of the wire saw, reducing the friction force, and it is beneficial to the cutting fluid to fully enter the cutting area and to exfoliate the debris quickly, reduce the surface roughness and improve the life of the wire saw.

\section{Conclusions}

In this paper, the experimental design of the process parameters and the output characteristics of the diamond wire saw in the process of cutting single crystal $\mathrm{SiC}$ is carried out by means of orthogonal experiment. Based on the optimized grey relational theory, the relationship between each process parameter and output characteristic index was analyzed, and the combination of process parameters to obtain the best processing quality and effect was analyzed.

On the basis of multi-objective grey correlation analysis, the influence degree of the multi-target output characteristics such as sawing force and surface roughness is obtained. The order of significance is the workpiece feed speed, wire saw speed, and workpiece rotation speed. And the best combination for obtaining the output characteristics is $\mathrm{B} 1 \mathrm{~A} 2 \mathrm{C} 2$, that is, the speed of the wire saw is $1.6 \mathrm{~m} / \mathrm{s}$, the workpiece feed speed is $0.025 \mathrm{~mm} / \mathrm{min}$, the workpiece rotation speed is $12 \mathrm{r} / \mathrm{min}$, the sawing force is $4.58 \mathrm{~N}$, and the surface roughness is $0.684 \mu \mathrm{m}$

At the same time, it is reflected in the complex and changeable system. Using the grey system theory to obtain the correlation factors which have significant influence on the output characteristic index and the optimal parameter combination to obtain the optimal output characteristic index, it has certain guiding and reference significance in the actual project.

\section{References}

1. M. ZHOU,J.Mater.Process.Technol.121,243251(2002)

2. E. Teomete,Arab. J. Sci. Eng.38(5),1209-1215(2013)

3. W. Clark, A Shih, C Hardin, et al,Int. J. Mach tool Manuf. 43(5),523-532(2003)

4. C. Hardin,J. Qu,A. Shih,Mater.Manuf.Process.19(2), 355-367(2004)

5. Y. Gao,P. Ge,T. Liu,Mater.Sci.Semicond.Process.56 ,106-114(2016)

6. Z. Khan,S. Kamaruddin,A. Siddiquee,Mater.Des.31( 6), $2925-2931(2010)$
7. H. Lu, C. Chang, N. Hwang, et al,J. Mater. Process. Technol.209(8),3808-3817(2009) 